\title{
Chaotic and regular motion around objects with quadrupolar and octupolar deformation.
}

\author{
Framsol López-Suspes ${ }^{1,2}$, Jerson I. Reina ${ }^{1,2}$ and Guillermo A. González ${ }^{2, *}$ \\ ${ }^{1}$ Departamento de Ciencias Básicas, Universidad Santo Tomás, Bucaramanga, Colombia \\ ${ }^{2}$ Escuela de Física, Universidad Industrial de Santander, Bucaramanga, Colombia
}

\begin{abstract}
Regular and chaotic test particle motion in axially symmetric gravitational fields due to bodies with quadrupolar and octupolar deformation are studied using Poincaré surfaces of section. We find that inclusion of the octupolar term induces a distortion in the KAM curves corresponding to regular trajectories, as well as an increasing in chaoticity. The fact the switching on of the octupolar moment it increases chaoticity and leads to the apparition of spindle torus, can be seen even in the case corresponding to oblate deformation, which commonly presents regular motion. Thus, the results here obtained are the generalization, for the case of Newtonian gravity, of those previously obtained by Heiss, W. D.,
\end{abstract} Nazmitdinov R. G. \& Radu, S. (1994) and Li, J. (1998) for harmonic oscillators.

Key words: Stellar dynamics; galaxies: kinematics and dynamics; chaotic dynamics.

Movimiento caótico y regular alrededor de objetos con deformación cuadrupolar y octupolar.

Resumen

Movimiento regular y caótico de partículas de prueba en campos gravitacionales axialmente simétricos debido a cuerpos con deformación cuadrupolar y octupolar es estudiado usando superficies de sección de Poincaré. Encontramos que la inclusión del término octupolar induce una distorsión en las curvas KAM correspondiente a trayectorias regulares, así como un aumento en la caoticidad. El hecho de que la activación del momento octupolar incremente la caoticidad y lleve a la aparición de regiones inestables, puede ser visto aún en el caso correspondiente a deformación oblata, la cual comúnmente presenta movimiento regular. Así, los resultados aquí obtenidos son la generalización, para el caso de gravedad Newtoniana, de los obtenidos previamente por Heiss, W. D., Nazmitdinov, R. G. \& Radu, S. (1994) y Li, J. (1998) para osciladores armónicos.

Palabras clave: Dinámica estelar; galaxias: cinemática y dinámica; dinámica caótica.

\section{Introduction}

As is suggested by a wide variety of observational evidences, many astrophysical objects can be modeled as axially symmetric bodies with prolate or oblate deformation, see Cooray, A. R. (2000), Davies, R. L. \& Birkinshaw, M. (1986), Fasano, G. \& Vio, R. (1991) and Moura, A. \& Letelier, P. S. (2000). As an example, it is known that the Earth has non vanishing quadrupolar and octupolar moments, as a consequence of its oblate shape (Boccaletti, D. \& Pucacco, G., 2004). Also, many galaxies with a large disc component can be assumed as axisymmetric oblate bodies with a large quadrupolar moment and, in some cases, with a significant octupolar deformation due to the remaining components like the halo (Helmi, A., 2004).

Likewise, there are galaxy clusters with a cigarlike shape (Cooray A. R., 2000) and many dwarf galaxies that can be considered as nearly axisymmetric prolate deformed objects (Ryden, B. S., 1996) with a non negligible octupolar moment. Also, some observational data on the sphericity of galaxy clusters suggest that triaxial galaxy models are more consistent with a prolate rather than an oblate distribution (Cooray A. R., 2000). Other triaxial galaxy models with only quadrupole approximations, have been built, e.g., by Schwarzschild, M. (1979) and Aquilano et al. (2007). On the other hand, the study of the analytical treatment of bifurcations of families of resonant thin tubes in axisymmetric galactic potentials was considered by Pucacco, G. (2009).

There are also models of oblate and prolate galaxies constructed through the dynamics of particles by numerical simulation of N-bodies (stars). These models of elliptical galaxies are self-consistent and can be found through the surfaces of section type box, tube and chaotic orbits, according to the effective potential (Contopoulos et al., 2002). Numerical models are variable, but always aimed to describe their morphology, surface luminosity profile, mass-light ratio, velocity dispersion profile, the line-of-sight velocity distribution curve and dark

\footnotetext{
*Correspondencia:

Guillermo A. González, guillermo.gonzalez@saber.uis.edu.co

Received: June 16, 2017

Accepted: August 30, 2017
} 
matter haloes (Capuzzo-Dolcetta et al., 2007, Jalali, M. A. \& Sobouti, Y., 1998, Jeon et al., 2009, Kimm, T. \& Yi, S. K., 2007, Sereno et al., 2006, Valluri et al., 2010, Zhenglu, J. , 2009).

Now, although the quadrupolar moment is usually considered to be the major deviation from the spherical symmetry, there are situations where the octupolar deformation play a comparable role, from the molecular level (Hamamoto et al., 1991, Frauendorf, S. \& Pashkevich, V. V., 1993) to the astrophysical context. There is evidence that dark matter clusters and groups may have substructure with a significant octupole deformation (Irwin, J. \& Shmakova, M., 2006), which can be revealed by studying the motion of freely falling objects.

In consequence, the motion of test particles around such stellar objects is a problem of wide physical interest. The case of attraction centers described by monopolar plus quadrupolar terms has been extensively studied, from a classical and relativistic standpoint, showing that the inclusion of external multipolar moments can induce chaos (Guéron, E. \& Letelier, P. S., 2001, 2002). In Newtonian gravity, as well as in general relativity, chaos can be found when the source has prolate deformation and the chaoticity grows by increasing the quadrupolar moment. On the other hand, it seems to be that the case corresponding to oblate deformation does not lead to chaotic motion, indeed for a very large quadrupolar deformation. However, inclusion of octupolar deformation can also induce chaos, as it was shown by Heiss, W. D., Nazmitdinov, R. G. \& Radu, S. (1994) and Li, J. (1998) in the case of an harmonic oscillator.

In this paper, we focus on astrophysical objects characterized by a non negligible octupolar moment in equilibrium (comparable with the quadrupolar deformation), so they can be described by a time independent potential. There are two important examples: axisymmetric galaxies with a prominent disc surrounded by a deformed halo, and a prolate deformed cluster of galaxies composed by an amount of dark matter with substructure (Irwin, J. \& Shmakova, M., 2006). In both cases, the dark component is responsible for the octupolar contribution and, as we shall show, it introduces new features in the orbital behavior of test particles.

We investigate the test particle motion in axially symmetric gravitational potentials that are the sum of monopolar, quadrupolar and octupolar terms (only external multipole moments will be considered). We analyze the effect that octupole deformation introduces in the phase-space structure associated to orbits. The paper is organized as follows. First, we define the form of the gravitational potential, present the Newtonian equations of motion, and show that the motion is restricted to a three-dimensional phase-space. Then, we perform the analysis by examining how the structure of the Poincaré surfaces of section is determined by the octupolar moment. So we find that, even in the case of oblate deformation, modest values of the octupolar moment induce significant changes in the phase-space structure. Finally, we summarize our main results.

\section{The Gravitational Potential}

Suppose that we have a known mass distribution and we want to find the potential or field outside the region where the source is. Consequently, the gravitational potential $\Phi$ that we are considering is a solution of the Laplace's equation (Arfken and Weber, 2005)

$$
\nabla^{2} \Phi=0 .
$$

Consider a distribution of mass $\rho\left(\vec{r}^{\prime}\right)$, confined to a region with $r<R$ (with $\Delta m$ the mass in a small volume $\Delta V=\Delta x \Delta y \Delta z)$ at the point $\vec{r} \prime$, thus $\Delta m=\rho\left(\vec{r}^{\prime}\right) \Delta V$. Let's expand the resulting gravitational potential for $r>R$ (Griffiths, D., 1999, Jackson, J. D., 1998)

$$
\Phi(\vec{r})=-G \int_{V} \frac{\rho\left(\vec{r}^{\prime}\right) d^{3} x^{\prime}}{\left|\vec{r}-\vec{r}^{\prime}\right|},
$$

where $G$ is the gravitational constant and $d^{3} x^{\prime}=d x^{\prime} d y^{\prime} d z^{\prime}$ is a three-dimensional volume element at $\vec{r}^{\prime}$.

To define the multipolar moments, we develop an expansion of the gravitational potential of an arbitrary localized mass distribution in powers of $\left|\vec{r}-\vec{r}^{\prime}\right|^{-1}$, that is

$$
\Phi(\vec{r}) \sim \sum_{n=0}^{\infty} \frac{1}{r^{n+1}} \int\left(r^{\prime}\right)^{n} P_{n}\left(\cos \theta^{\prime}\right) \rho\left(\vec{r}^{\prime}\right) d^{3} x^{\prime},
$$

where $\theta^{\prime}$ is the angle between $\vec{r}$ and $\vec{r}^{\prime}$, and $P_{n}$ is the Legendre polynomial of order $n$. The above expression in explicit form is

$$
\Phi(\vec{r}) \sim \frac{1}{r} \int \rho\left(\vec{r}^{\prime}\right) d^{3} x^{\prime}+\frac{1}{r^{2}} \int r^{\prime} \cos \theta^{\prime} \rho\left(\vec{r}^{\prime}\right) d^{3} x^{\prime}+\cdots
$$

The term $n=0$ (monopole), is the mass of the source, $n=1$ should be the mass dipole, the third term is the mass quadrupole and so on (according to $2^{n}$ poles). However, the mass dipole and the mass quadrupole do not have an analog in gravitation because there is not a counterpart $-M$ to form the mass dipole. Evidently, the potential of a quadrupole goes like $1 / r^{3}$ and as $1 / r^{4}$ in an octupole, as we might have anticipated.

The expression that includes all the terms of exact development of the gravitational potential considering cylindrical symmetry can be written as (Fernandez, A. , 2005)

$$
\Phi(\vec{r})=\frac{-G M}{r}\left(1-\sum_{n=2}^{\infty} \frac{C_{n}}{r_{n}} P_{n}(\cos \theta)\right),
$$

where the constants $C_{n}$ are related with the multipole moments of mass, and $M$ is the mass of the source. Moreover, the terms with $n$ odd are asymmetric respect to the north and the south, e.g. $C_{3}$ is related with objects in shape of pear (octupole). The term corresponding to $n=2$ is associated with the flattened or elongated deformation of the objects.

\section{The Equations of Motion}

Consider a test particle moving in the axially symmetric gravitational field generated by a stellar body with quadrupolar and octupolar deformation. In cylindrical coordinates $(R, z, \varphi)$, the potential has the form (Binney and Tremaine, 2008)

$$
\Phi(R, z)=-\frac{\alpha}{\sqrt{R^{2}+z^{2}}}-\frac{\beta\left(2 z^{2}-R^{2}\right)}{2\left(R^{2}+z^{2}\right)^{\frac{5}{2}}}-\frac{\gamma\left(2 z^{3}-3 z R^{2}\right)}{2\left(R^{2}+z^{2}\right)^{\frac{7}{2}}},
$$

where $\alpha$ is the monopole term, which is equals to $G m$, with $m$ the total mass of the source and $G$ the gravitational constant. The quadrupolar term, denoted by $\beta$, usually represents the major deviation from spherical symmetry. In particular, if $\beta>0$ the source has prolate deformation and if $\beta<0$ we have the case corresponding to oblate deformation. The octupolar moment $\gamma$ describes the asymmetry of the source with respect to the equatorial plane, i.e. its "shape of pear" deformation. 
Both $\beta$ and $\gamma$ are related to the source's density $\rho(R, z)$ through the equations (Binney and Tremaine, 2008)

$$
\begin{aligned}
& \beta=2 \pi G \int_{0}^{\infty} r^{\prime 4} d r^{\prime} \int_{0}^{\pi} d \theta^{\prime} \sin \theta^{\prime} P_{2}\left(\cos \theta^{\prime}\right) \rho\left(r^{\prime}, \theta^{\prime}\right), \\
& \gamma=2 G \pi \int_{0}^{\infty} r^{\prime 5} d r^{\prime} \int_{0}^{\pi} d \theta^{\prime} \sin \theta^{\prime} P_{3}\left(\cos \theta^{\prime}\right) \rho\left(r^{\prime}, \theta^{\prime}\right),
\end{aligned}
$$

where we have used spherical coordinates $r=\sqrt{R^{2}+z^{2}}$, $\cos \theta=z / \sqrt{R^{2}+z^{2}}$ and $P_{l}$ denotes the Legendre polynomial of order $l$.

The physical interest to analyze other terms of the multipolar expansion (quadrupolar and octupolar terms), is based in that they are directly related to the astrophysical objects in rotation or having a pear-shaped deformation, or both. When celestial objects have a non-negligible rotation, this produces an oblate deformation between the north and south poles, i.e. flattened at the poles as is the case of Earth. The contribution of the mass quadrupolar is extremely important for motion of test particle close to celestial object or artificial satellites close to Earth. Due to change of mass density, the astrophysical objects tend to be deformed, thus in the special case of Earth the octupolar term is necessary due to his pear form. In the context of general relativity, the mass quadrupole moment is important, due to that if this varies over time, it can cause gravitational radiation, equal to the electromagnetic radiation generated by oscillating electric or magnetic dipoles and higher order multipoles. Although, only quadrupole and higher moments (octupole) are known to can radiate gravitationally.

The motion of a test particle in a gravitational field described by (1), obeys the relations (López-Suspes, F. \& González, G. A., 2013)

$$
\begin{aligned}
\dot{R} & =V_{R}, \\
\dot{z} & =V_{z}, \\
\dot{V}_{R} & =-\frac{\partial}{\partial R} \Phi_{e f f}(R, z), \\
\dot{V}_{z} & =-\frac{\partial}{\partial z} \Phi_{e f f}(R, z),
\end{aligned}
$$

where $\Phi_{e f f}(R, z)$ is the effective potential, given by

$$
\Phi_{e f f}(R, z)=\Phi(R, z)+\frac{\ell^{2}}{2 R^{2}} .
$$

Here, $\ell=R^{2} \dot{\varphi}$ is the axial specific angular momentum that is conserved as a consequence of the axial symmetry. The second integral of motion is the total specific energy

$$
\mathcal{E}=\frac{1}{2}\left(V_{R}^{2}+V_{z}^{2}\right)+\Phi_{\text {eff }}(R, z) .
$$

According to eqs. (4)-(9), the motion is restricted to a three dimensional phase space $\left(R, z, V_{R}\right)$. This fact enable us to introduce the Poincaré surfaces of section method, in order to investigate the trajectories of test particles. Note that the orbit of particle is confined to the plane defined by the effective potential, known as meridional plane. The numerical integration is performed through the Runge-Kutta method of fourth order and by checking that energy is conserved within a tolerance of $10^{-10}$. The initial conditions necessary to solve the differential equations system of motion can be obtained as follows: we select the conditions for the initial positions, i.e.
$R(t=0)=R_{0}, z(t=0)=z_{0}$, according to the contour of the effective potential, which are obtained when total specific energy is equal to a constant. Then an initial condition is fixed for the vertical speed $z(t=0)=z_{0}$, and finally we can determine from the constraint (9), the initial condition for the radial velocity $\dot{R}(t=0)=\dot{R}_{0}$.

\section{Chaos Induced by Octupolar Deformation}

Currently exist reports on the chaotic behavior of test particles moving around gravitational fields produced for superposition of monopole and oblate quadrupole (Letelier et al., 2011). Likewise there are numerical evidences that orbits of particles moving around a monopole plus an prolate quadrupole are chaotic (Guéron, E. \& Letelier, P. S., 2001). In Newtonian gravity has been studied chaotic behaviors through of test particle of some configurations of matter that may have an interest in astrophysics, like the investigation on the integrability of oblique orbits of the test particles around gravitational fields produced for superposition of black-hole and thin disk (Saa, A. \& Venegeroles, R., 1999), or around gravitational fields generated of a monopole and a thick disk (Saa, A., 2000), and gravitational field with dipoles (Ju-Hua, C. \& Yong-Jiu, W., 2003). Accordingly, we considered bodies with prolate or oblate deformation plus octupolar moment, we presented some surface of section for different values of octupolar term, one can confirmed that the parameter of "shape-of-pear" deformation induced a chaotic behavior.

In figure 1 we plot a typical $z=0$ surface of section corresponding to motion of a test particle in presence of a gravitational field due to a prolate deformed source, whose octupolar moment vanishes. We note a central and lateral regular regions composed by ring tori (ring KAM curves). They are enclosed by a chaotic region containing two small resonant islands near its top and bottom edges. In figure 1(a) we show Poincaré section for some orbits with $\ell=0.9$ and $\mathcal{E}=-0.4$, in a potential with $\alpha=1, \beta=0.3$ and $\gamma=0$. In figure $1(\mathrm{~b})$ we change the value of the octupolar moment $(\gamma=0.02)$, maintaining the same prolate deformation. The resulting surface of section presents a more prominent chaotic region, since in this case the outer zones of resonant islands have overlapped. The regular regions now contains "spindle" torus. They can be viewed clearly in the central region and scarcely insinuated in the lateral zone. In figure 1(c), as a consequence of increase the octupolar moment to $\gamma=0.04$, the chaotic region is more prominent (the lateral regular zone has disappeared), as well as the central spindle KAM curves. In this path we keep the values $\ell=0.9, \mathcal{E}=-0.4, \alpha=1, \beta=0.3$. In this figure, we can see as the inclusion of the octupolar term affect the structure of phase space. We can see as the stable regions presents now a stochastic behavior.

In figure 2 we show the effect caused by the progressive rise in the octupolar deformation, starting from a regular prolate situation. In particular, we show the Poincaré section for $\ell=1.1, \mathcal{E}=-0.32, \alpha=1, \beta=0.2$ and the cases (a) $\gamma=0$, (b) $\gamma=0.02$, (c) $\gamma=0.04$, and (d) $\gamma=0.06$. In each one, the orbits are generated by the initial conditions (i) $z=0, R=0.91$, $V_{R}=0$, (ii) $z=0, R=0.78, V_{R}=0$, and (iii) $z=0, R=1.16$, $V_{R}=0.18$.

In figure 3 we display the trajectories in the meridional plane with initial conditions $z=0, R=0.78, V_{R}=0$, and the same parameters considered above. Again we have the cases (a) $\gamma=0$, (b) $\gamma=0.02$, (c) $\gamma=0.04$, and (d) $\gamma=0.06$. The fact 


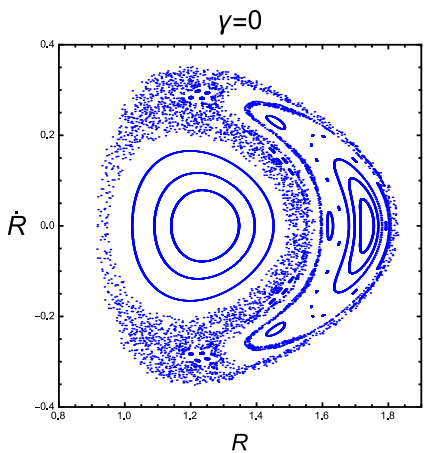

(a)

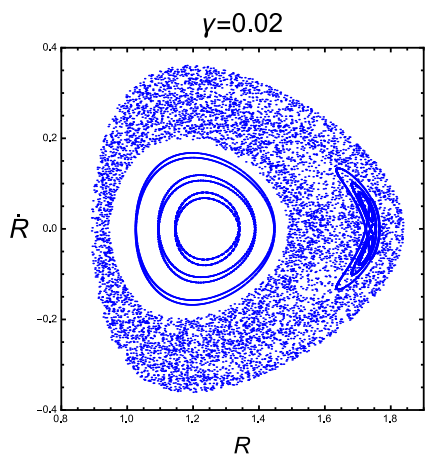

(b)

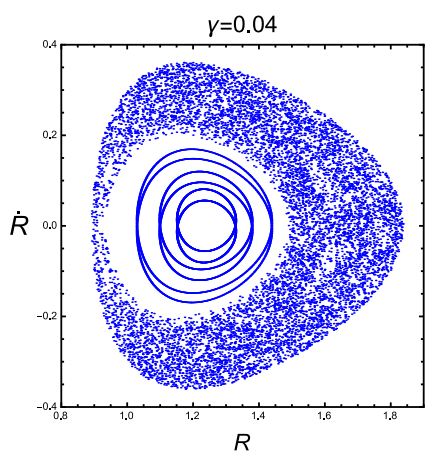

(c)

Figure 1. Surfaces of section for some orbits with $\ell=0.9, \mathcal{E}=-0.4$, in a potential characterized by $\alpha=1, \beta=0.3$ (prolate deformation), and (a) $\gamma=0$, (b) $\gamma=0.02$, and (c) $\gamma=0.04$. In (c), we have a prominent chaotic zone enclosing three "spindle" KAM curves.

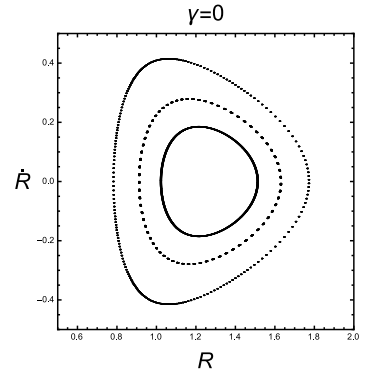

(a)

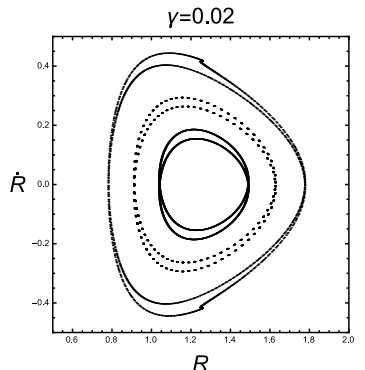

(b)

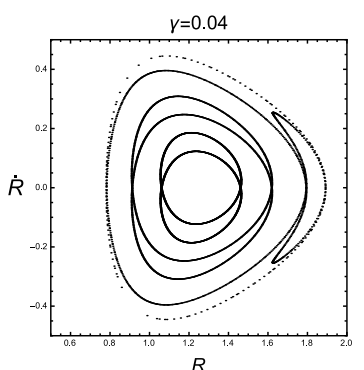

(c)

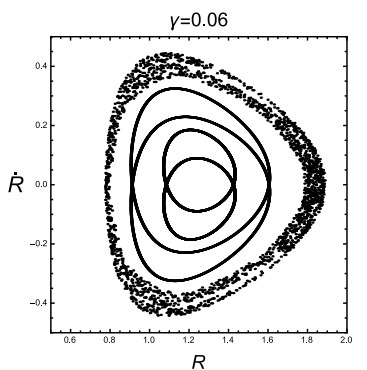

(d)

Figure 2. Surfaces of section for $\ell=1.1, \mathcal{E}=-0.32, \alpha=1, \beta=0.2$ and (a) $\gamma=0$, (b) $\gamma=0.02$, (c) $\gamma=0.04$, and (d) $\gamma=0.06$. In each case, they are generated by three orbits with initial conditions (i) $z=0, R=0.91, V_{R}=0$, (ii) $z=0$, $R=0.78, V_{R}=0$, and (iii) $z=0, R=1.16, V_{R}=0.18$.

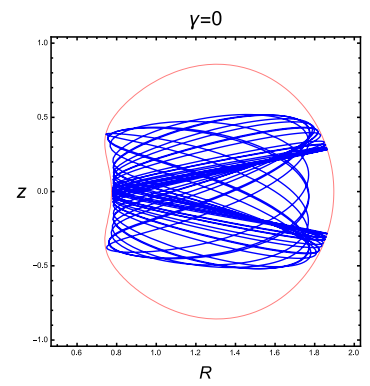

(a)

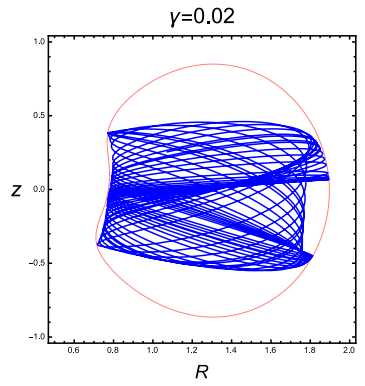

(b)

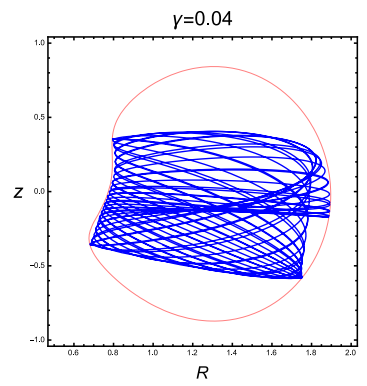

(c)

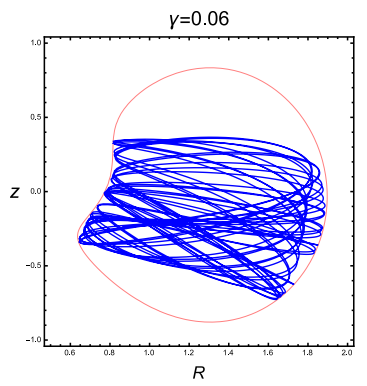

(d)

Figure 3. Orbits in the meridional plane with initial conditions $z=0, R=0.78, V_{R}=0$ and the same parameters considered above. Again we have the cases (a) $\gamma=0$, (b) $\gamma=0.02$, (c) $\gamma=0.04$, and (d) $\gamma=0.06$.

the switching on of the octupolar moment it increases chaoticity and leads to the apparition of spindle torus, can be seen even in the case corresponding to oblate deformation, which commonly presents regular motion. In fact, some authors show numerical evidence that particles moving around a monopole plus an oblate quadrupole are not chaotic, however this result has been recently corrected by Letelier et $\boldsymbol{a l}$. (2011).

Finally, in figure 4 we show the transition from regularity to chaos by increasing $\gamma$. With $\mathcal{E}=-0.32, \ell=1.1$ and $\beta=-0.2$, we start from (a) $\gamma=0$, (b) $\gamma=0.02$, (c) $\gamma=0.04$, and (d) $\gamma=0.06$. The case (a) corresponds to a regular motion, the case (b) is also regular but the KAM curves has been distorted to spindles. In (c) the distortion is more prominent and finally, in (d), we note the apparition of a chaotic region enclosing the spindles.

Figure 5 shows the contours of the gravitational potential, in all paths we consider $\alpha=1$. In this plot we present the deformation of the source due to changes in the multipole moments of mass. To show the oblate deformation we consider $\beta=-0.3$ and for prolate deformation we chose $\beta=0.3$ with octupolar 


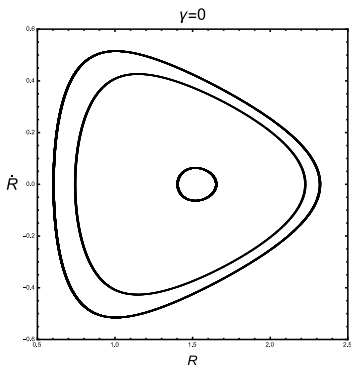

(a)

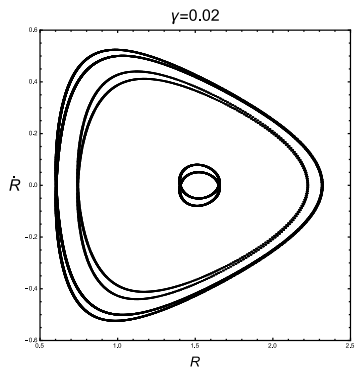

(b)

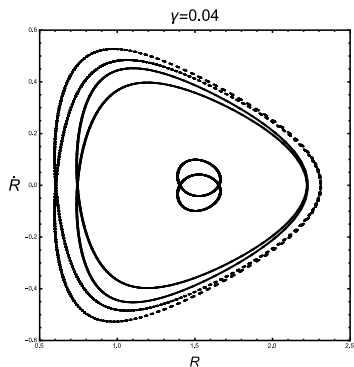

(c)

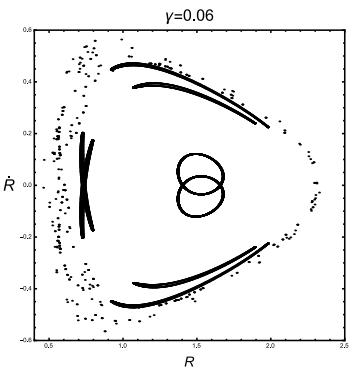

(d)

Figure 4. Surfaces of section for some orbits with $\ell=1.1, \mathcal{E}=-0.32$, in a potential characterized by $\alpha=1, \beta=-0.2$ and (a) $\gamma=0$, (b) $\gamma=0.02$, (c) $\gamma=0.04$, and (d) $\gamma=0.06$. In each case, they are generated by three orbits with initial conditions (i) $z=0, R=1.4, V_{R}=0$, (ii) $z=0, R=0.74, V_{R}=0$, and (iii) $z=0, R=0.601, V_{R}=0.4$.

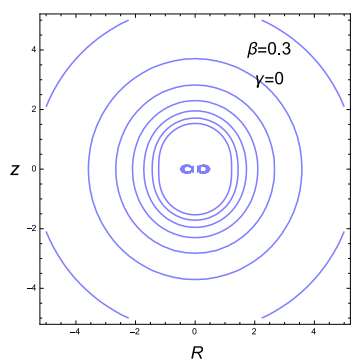

(a)

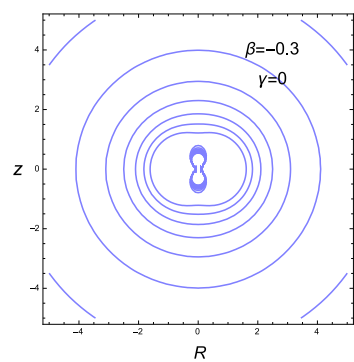

(b)

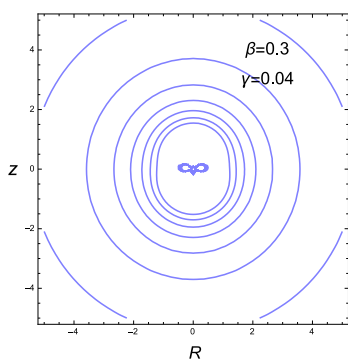

(c)

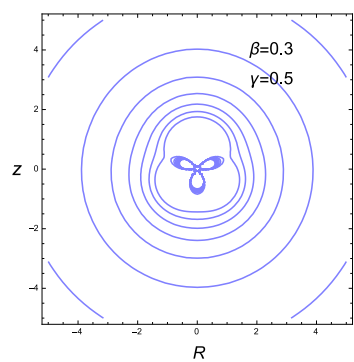

(d)

Figure 5. Contours of the effective potential. We consider the parameters $\alpha=1, \beta= \pm 0.3$ and $\gamma=0,0.04$, and 0.5 ( "shape-of-pear").

moment constant $(\gamma=0)$. Note that the octupolar deformation of the source begins to be appreciated for values close to $\gamma=0.5$, however the chaos can be observed for small values of the octupolar moment: $\gamma=0.02$ and $\gamma=0.04$.

Observe that figure 1, figure 2 and figure 4 represent the superposition of several solutions of the autonomous system of differential equations (4) - (7). Each of the trajectories is obtained by means of different initial conditions, in agreement with the KAM theorem: different initial conditions of the integrable Hamiltonian system will trace different invariant tori in the phase space. When the trajectories in the figure 1, figure 3(a), and figure 4(a) intersect them do not represent the same solution, every set of initial conditions is a torus in the structure of phase space

\section{Concluding Remarks}

From a classical point of view, octupolar deformation in astrophysical objects can introduce significant modifications to the phase-space structure corresponding to test particles moving around prolate or oblate centers of attraction. Apart from an increasing in the chaoticity, the apparition of spindle torus in regular regions is a remarkable effect caused by the asymmetry of the source with respect to its equatorial plane. A larger equatorial asymmetry involves more distorted spindle KAM curves and more prominent stochastic regions in the phase-space (See figure 1 for the prolate situation, $\beta=0.3$ ). This fact carries dramatic consequences in the case of oblate deformed sources, which usually are associated with regular motion. Here, chaos emerges once we switch on the octupole moment. The results here obtained are then the generalization, for the case of Newto- nian gravity, of those previously obtained by Helmi, A. (2004) and $\mathbf{L i}, \mathbf{J}$. (1998) for harmonic oscillators. In summary, we show that may appear stochastic zones in the surfaces of section due only to the existence of "shape-of-pear" deformation in regions that previously were stable.

\section{Contribution of the authors}

All the authors have contributed in various degrees to the conception of the work, to the research concept, to the analytical methods used, to the drafting of the article, to the critical revision of the article and to the final approval of the version to be published.

\section{Acknowledgments}

This research was partially supported by VIE-UIS, under grant number 1838, and COLCIENCIAS, Colombia, under grant number 8840. JIR and FLS wants to thank the support from Centro de Investigaciones, Universidad Santo Tomás, Bucaramanga.

\section{Conflict of interest}

The authors declare that they have no conflict of interest.

\section{References}

Arfken, G. and Weber, H. (2005). Mathematical Methods for Physicists. 6th ed. Academic Press.

Aquilano, R. O., Muzzio, J. C., Navone, H. D. \& Zorzi A. F. (2007). Orbital structure of self-consistent triaxial stellar systems. Celestial Mech. Dynam. Astronom. 99, 307. 
Binney, J. and Tremaine, S. (2008). Galactic Dynamics. 2nd ed. Princeton University Press.

Boccaletti, D. \& Pucacco, G. (2004). Theory of Orbits, Volume 1, Springer. Third edition.

Capuzzo-Dolcetta, R., Leccese, L., Merritt, D. \& Vicari, A. (2007). Self-consistent models of cuspy triaxial galaxies with dark matter halos. Ap. J. 666, 165.

Cooray A. R. (2000). Galaxy clusters: oblate or prolate? Mon. Not. R. Astron. Soc. 313, 783.

Contopoulos, G., Voglis, N. \& Kalapotharakos, C. (2002). Order and Chaos in Self-Consistent Galactic Models. Celestial Mech. Dynam. Astronom. 83, 191.

Davies, R. L. \& Birkinshaw M. (2010). NGC 4261 - A prolate elliptical galaxy. Ap. J. 303, L45.

Fasano, G. \& Vio, R. (1991). Apparent and true flattening distribution of elliptical galaxies. Mon. Not. R. Astron. Soc. 249, 629.

Fernandez, A. (2005). Dinámica Clásica. 2da edición. Fondo de Cultura Económica USA.

Frauendorf, S. \& Pashkevich, V. V. (1993). Shapes of Na Clusters. Z. Phys. D 26, 98.

Griffiths, D. (1999). Introduction to Electrodynamics. Third edition. Prentice Hall, New Jersey.

Guéron, E. \& Letelier, P.S. (2001). Chaos in pseudo- Newtonian black holes with halos. A \& A 368, 716.

Guéron, E. \& Letelier, P.S. (2002). Geodesic chaos around quadrupolar deformed centers of attraction. Phys. Rev. E 66, 046611.

Hamamoto, I., Mottelson, B., Xie, H. \& Zhang, X. Z. (1991). Shell-structure and octupole instability in fermion systems. Z Phys. D 21, 163

Heiss, W. D., Nazmitdinov R. G. \& Radu, S. (1994). Chaos in axially symmetric potentials with octupole deformation. Phys. Rev. Lett. 72, 2351.

Helmi, A. (2004). Is the dark halo of our Galaxy spherical. Mon. Not. R. Astron. Soc. 351, 643.

Irwin, J. \& Shmakova, M. (2006). Observation of small-scale structure using sextupole lensing. Ap. J. 645, 17.

Jackson, J. D. (1998). Classical Electrodynamics. Third Edition. Wiley Editorial.

Jalali, M. A. \& Sobouti, Y. (1998). Some Analytical Re- sults in Dynamics of Spheroidal Galaxies. Celestial Mech. Dynam. Astronom. 70, 255.
Jeon, M., Kim, S. S. \& Ann, H. B. (2009). Galactic Warps in Triaxial Halos. Ap. J. 696, 1899.

Ju-Hua, C. \& Yong-Jiu, W. (2003). Chaos in a gravitational field with dipoles. Chin. Phys. 12, 836.

Kimm, T. \& Yi, S. K. (2007). Intrinsic axis ratio distribution of early-type galaxies from the sloan digital sky survey. Ap. J. 670, 1048.

Letelier P.S., Ramos-Caro J. \& López-Suspes F. (2011). Chaotic motion in axially symmetric potentials with oblate quadrupole deformation. Phys. Lett. A 375, 3655.

Li, J. (1998). The stability of trajectories in an axially symmetric potential with octupole deformation. Phys. G: Nucl. Part. Phys. $24,1021$.

López-Suspes, F. \& González, G. A. (2013). Thick Disks with newtonian multipolar moments. Rev. Acad. Colomb. Cienc. Ex. Fis. Nat. 37, 301.

Moura, A. \& Letelier, P. S. (2000). Chaos and fractals in geodesic motions around a nonrotating black hole with halos. Phys. Rev. E 61, 6506.

Pucacco, G. (2009). Resonances and bifurcations in axisymmetric scale-free potentials. Mon. Not. R. Astron. Soc. 399, 340.

Ryden, B. S. (1996). The Intrinsic Shapes of Stellar Systems. Ap. J. $461,146$.

Saa, A. \& Venegeroles, R. (1999). Chaos around the superposition of a black-hole and a thin disk. Phys. Lett. A 259, 201.

Saa, A. (2000). Chaos around the superposition of a monopole and a thick disk. Phys. Lett. A 269, 204.

Schwarzschild, M. (1979). A numerical model for a triaxial stellar system in dynamical equilibrium. Ap. J. 232, 236.

Sereno, M., De Filippis, E., Longo, G. \& Bautz, M.W. (2006). Measuring the three-dimensional structure of galaxy clusters. II. Are clusters of galaxies oblate or prolate. Ap. J. 645, 170.

Valluri, M., Debattista, V.P., Quinn, T. \& Moore, B. (2010). The orbital evolution induced by baryonic condensation in triaxial haloes. Mon. Not. R. Astron. Soc., 403, 525.

Zhenglu, J. (2009). Dynamical modelling of the elliptical galaxy NGC 2974. Celestial Mech. Dynam. Astronom. 103, 31. 